\title{
EVOLUTION OF SURFACE ROUGHNESS DURING CVD GROWTH
}

E. CHASON*, T.M. MAYER*, D.P. ADAMS*, H. HUANG**, T.DIAZ DE LA RUBIA**, G. GILMER $^{\dagger}$, B. K. KELLERMAN ${ }^{\dagger \dagger}$

*Sandia National Laboratories, Albuquerque, NM

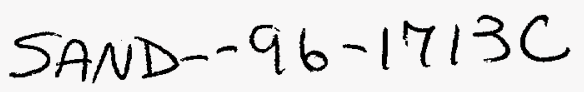

**Lawrence Livermore National Laboratory, Livermore, CA

${ }^{\dagger}$ Lucent Technologies, Murray Hill, NJ

${ }^{+\dagger}$ MEMC Electronic Materials, St. Peters, MO

RECEIVED

ABSTRACT

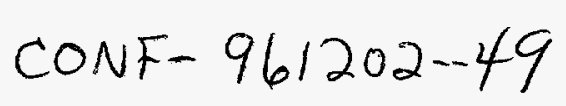

JAN 061997

OSTI

Monte Carlo simulations of physical and chemical vapor deposition are used to study roughening kinetics of films that grow by nucleation and coalescence of clusters. The effects of interlayer transport, preferential dissociation of molecular precursors and energetic differences between the clusters and the substrate are examined.

\section{INTRODUCTION}

The kinetics of surface roughening are intimately related to fundamental surface processes such as adatom diffusivity, island nucleation, and attachment/detachment kinetics. Because these processes all occur simultaneously, it is difficult to determine their individual effects on the evolution of the roughness. In this work, we discuss the results from kinetic Monte Carlo simulations of film deposition that have been performed to understand the fundamental processes that determine surface roughness evolution. Three separate mechanisms that contribute to surface roughness are considered: 1) interlayer transport, 2) preferential sticking or dissociation and 3) and energetic differences between the clusters and the substrate.

The computer simulations are motivated by X-ray reflectivity (XRR) measurements of the evolution of the surface roughness during chemical vapor deposition (CVD) of $\mathrm{Fe}$ on $\mathrm{Si}(001)$ surfaces [1]. Fe was deposited using a $\mathrm{Fe}(\mathrm{CO})_{5}$ molecular precursor at pressures in the range of $10^{-7}-10^{-8}$ torr and growth temperatures of $100-255^{\circ} \mathrm{C}$. XRR enables simultaneous measurement of the film thickness and surface roughness. The measured thickness vs. exposure is shown in fig. 1a. Unlike physical vapor deposition, the thickness is not linear with the exposure because of the process of nucleating Fe clusters on the Si surface. Preferential dissociation of the precursor on the Fe clusters causes the growth rate to increase with exposure until the steady state growth of Fe on Fe occurs. The surface roughness vs. deposited film thickness is shown in fig. $1 \mathrm{~b}$. The roughness increases very rapidly with thickness at low temperature, so that the roughness initially grows faster than the layer thickness. After the maximum roughness is reached, there is also a sudden decrease in the roughness associated with coalescence of the individual clusters into a continuous film.

\section{MONTE CARLO SIMULATIONS}

The Monte Carlo simulation have been discussed previously [2,3] so only a brief description will be given here. The main concept behind the simulations is the identification of a series of surface processes that happen during growth, e.g., deposition, surface diffusion, diffusion over step edges, cluster nucleation, attachment and detachment from islands. A transition rate is then associated with each of these processes, and the surface processes are chosen to occur with a probability proportional to their relative rates. These rates may depend on the local atomic coordination so that, for instance, the barrier for detachment from an $n$-coordinated site is equal to $E_{o}+n \delta \varepsilon$ where $E_{o}$ is the activation energy for adatom diffusion on the free surface and $\delta \varepsilon$ is an addition bond-energy term making the activation energy proportional to the number of neighbors. 


\section{DISCLAIMER}

This report was prepared as an account of work sponsored by an agency of the United States Government. Neither the United States Government nor any agency thereof, nor any of their employees, make any warranty, express or implied, or assumes any legal liability or responsibility for the accuracy, completeness, or usefulness of any information, apparatus, product, or process disclosed, or represents that its use would not infringe privately owned rights. Reference berein to any specific commercial product, process, or service by trade name, trademark, manufacturer, or otherwise does not necessarily constitute or imply its endorsement, recommendation, or favoring by the United States Government or any agency thereof. The views and opinions of authors expressed herein do not necessarily state or reflect those of the United States Government or any agency thereof. 

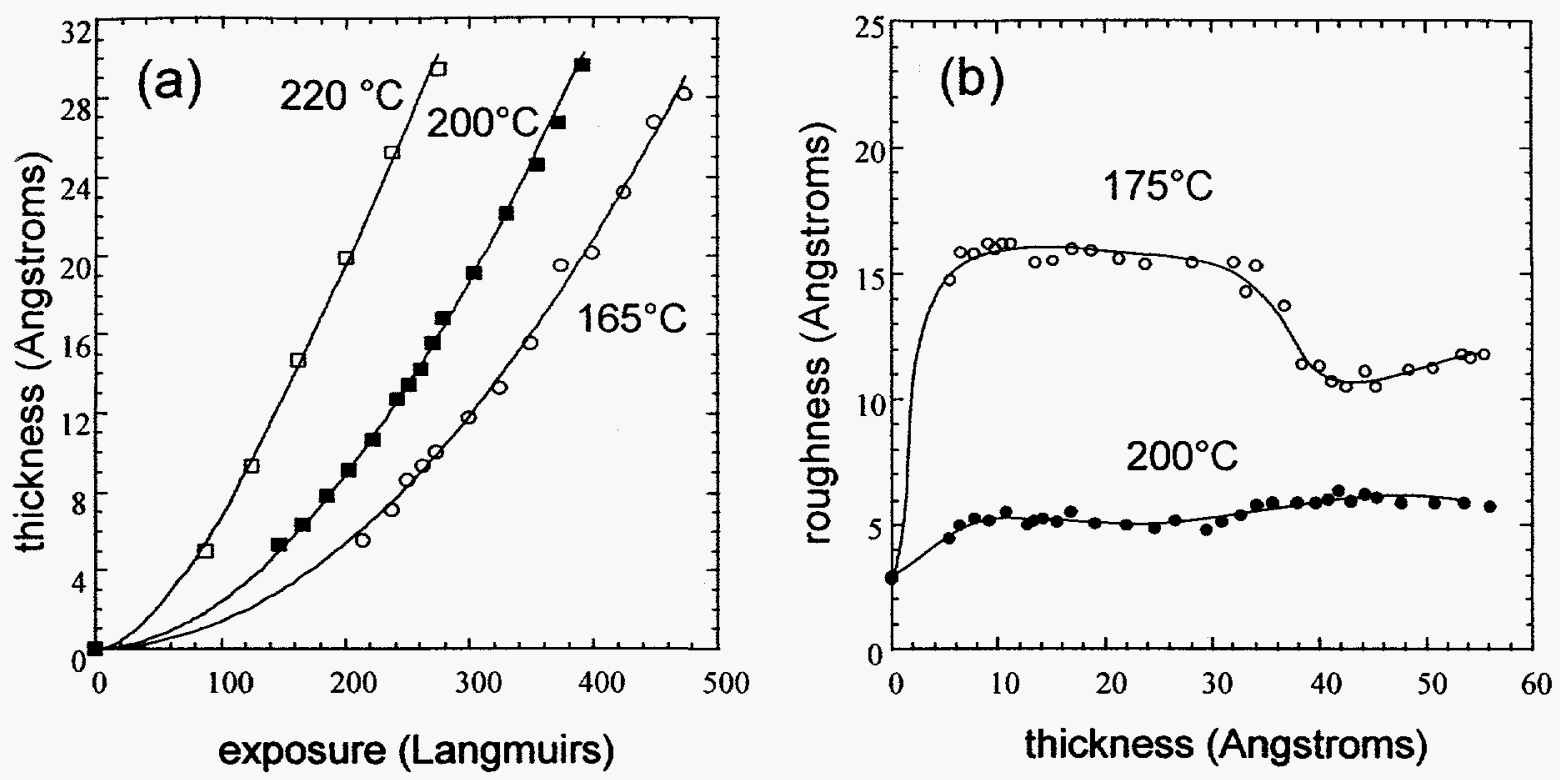

Figure 1. (a) Evolution of film thickness with exposure to $\mathrm{Fe}(\mathrm{CO})_{5}$ during $\mathrm{CVD}$ of $\mathrm{Fe}$ on $\mathrm{Si}$ at temperatures indicated on figure. (b) Evolution of surface roughness with film thickness at temperatures indicated on figure.

In the simulations of CVD growth, we allow there to be 2 species: the adatom, which can diffuse and cluster as described above, as well as a mobile precursor which impinges on the surface at a rate determined by the growth pressure. This precursor is given the additional properties of being able to diffuse on the surface, desorb from the surface and dissociate on the surface (after which it becomes an adatom). The dissociation rate is allowed to depend on the local environment so that the rate of dissociation on the Si surface, at the edge of clusters and on top of clusters can be varied independently. The parameters used for the various surface processes in the CVD simulations are given in table 1. Parameters for the polycrystalline simulations (Al on amorphous substrate) will be published elsewhere [4].

\section{ROUGHENING BEHAVIOR}

The goal of this work is to understand what is required to obtain the very rapid initial roughening and the subsequent decrease in the roughness as the film gets thicker. In the following sections, we consider three different atomistic mechanisms and how they contribute to the surface roughness. The first is the

deposition/arrival rate

diffusion: on terrace (0-coordination) across step edge

detachment (from $\mathbf{n}$-coordinated site)

desorption

dissociation of precursor: on substrate

at cluster edge

on top of cluster

sticking coefficient:

on $\mathrm{Si}$ adatoms

0

1.0

1.2

$1.0+0.2 * \mathrm{n}$ precursor

0.5 monolayers $/ \mathrm{s}$

0.1

2

0.35

0.35

0.05

0

.001

on Fe cluster

Table 1. Parameters used in CVD growth simulations (all energies in eV). 
presence of a barrier at the step edges that prevents transport of adatoms between layers. Next, we consider the effect of preferential dissociation of the precursor molecule on the nucleated Fe clusters. Finally, we look at the effects of energetic differences between the cluster and the substrate as well as between clusters with different orientations (grain boundary energies).

\section{Effect of Interlayer Transport on Roughness}

The effect of interlayer transport on the evolution of roughness can be understood analytically, as has been discussed by Cohen et al [5]. If we define $\theta_{n}$ as the exposed coverage of the $n^{\text {th }}$ layer, and $k_{o}$ as the deposition rate, then we can derive a set of expressions that describes how the occupation of each layer evolves with time. In the absence of any interlayer transport (i.e., an infinite barrier at the step edges preventing atoms from changing layers), the rate at which the $n^{\text {th }}$ layer decreases its area by being covered up with new atoms is proportional to the area of the nth layer. The $\mathrm{n}^{\text {th }}$ layer also increases its area when atoms land on the $(n-1)^{\text {th }}$ layer. Solving the series of equations for the occupancy of the $n^{\text {th }}$ layer with time gives $\theta_{n}=\exp \left(-k_{0} t\right)\left(k_{0} t\right)^{n} / n$ ! which is simply a Poisson distribution of layer occupations. Therefore, the average layer height, $<n>$, is equal to $k_{0} t$ and the RMS roughness, $\sigma$, is equal to $\left(k_{0}\right)^{1 / 2}$. This results indicates that no matter how large the barrier is to interlayer transport, the roughness can never grow faster than the square root of the layer thickness. In addition, the RMS roughness is independent of the diffusion in the layer (intra-layer transport). Monte Carlo simulations show that changing the intra-layer diffusivity can dramatically alter the morphology from a very random surface at low diffusivity to large mounds at high diffusivity. However, even though these surfaces look very different because of their vastly different in-plane correlation, they still have the same RMS roughness.

\section{Effect of Preferential Dissociation}

In this section, we consider the effect of preferential dissociation of the precursor molecule on the surface of the Fe cluster relative to the substrate. In order to study the evolution of roughness with interlayer transport and preferential dissociation, we use Monte Carlo simulations containing two species (adatom and precursor) as described above.

The evolution of the film thickness with time is shown in fig. 2a. The growth kinetics display a non-linear behavior similar to that observed experimentally. The nucleation time before steady-state growth is achieved is dependent on temperature, and gets longer for lower temperatures. The evolution of the roughness with thickness is shown in fig. $2 \mathrm{~b}$. The addition of a mechanism for preferential dissociation leads to much more rapid roughening than the Poisson distribution in the previous section. The maximum surface roughness is larger for the low temperature growth.

To understand the temperature dependence of the surface roughness evolution, we must consider the balance between various surface processes. Because the dissociation rate is much larger on the clusters than on the substrate (by a factor of 1000 in these simulations), once the islands nucleate they accumulate more $\mathrm{Fe}$ adatoms very rapidly. The rate at which the islands grow laterally (to cover the substrate) is primarily determined by interlayer transport on the island. The islands fill in because of precursor molecules dissociating on the cluster and then diffusing down to the substrate as $\mathrm{Fe}$ atoms. In the absence of interlayer transport, the islands grow laterally by dissociation of precursor molecules at the edge of the islands, but this is a much slower process under the conditions discussed here.

Because the activation energy used in these simulations for interlayer transport of adatoms is 0.2 ev larger than for diffusion of adatoms on the same surface, the interlayer transport is significantly larger at higher temperatures. The enhancement of the lateral growth of the islands can be seen in the time it takes for islands to coalesce on the surface. The number of individual Fe clusters on the surface as a function of time and temperature is shown in fig. 3. For the case of growth at $250 \mathrm{C}$, the clusters nucleate more rapidly, reaching a maximum of 64 clusters. At lower temperatures, the nucleation period is longer, and the maximum number of clusters is slightly smaller. Most significant, however, is that it 

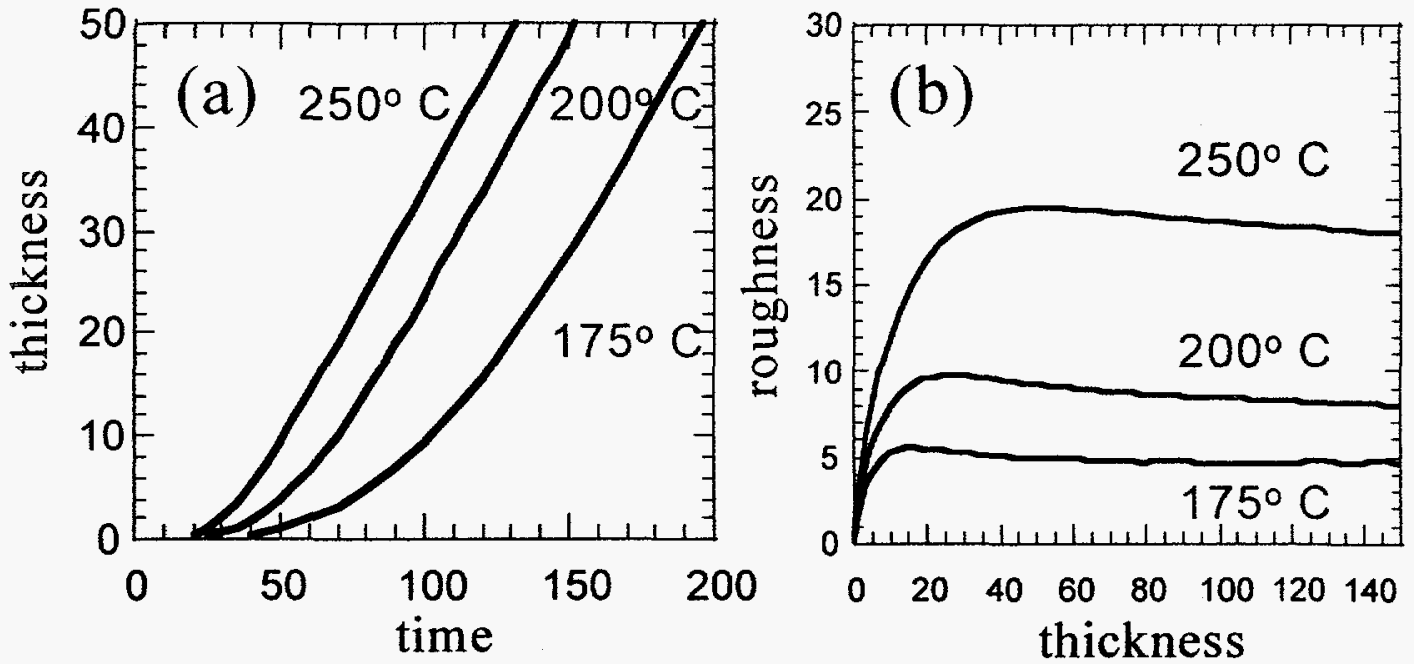

Figure 2. (a) Evolution of film thickness with time from simulations. (b) Evolution of roughness with thickness from simulations.

takes a much longer time before the clusters come together. If we define the coalescence time as the FWHM of the distributions in fig. 3 (i.e., the time between nucleation of half the maximum number ofclusters and disappearance of half the clusters) we see that the coalescence time increases from $12 \mathrm{~s}$ at $250 \mathrm{C}$ to $67 \mathrm{~s}$ at $175 \mathrm{C}$. Since the activation for dissociation on the clusters is small, and therefore the rate does not depend strongly on the temperature, the amount of time that the clusters grow before coming together determines how large the maximum roughness is.

These simulations with preferential dissociation of the precursor on clusters can explain why the roughness grows so rapidly, but the degree of smoothing after the maximum roughness is reached is smaller in the simulations than in the experiments. One possible explanation for this is that there is no barrier to adatoms diffusing off of the cluster and onto the subtrates in the results discussed above. In the

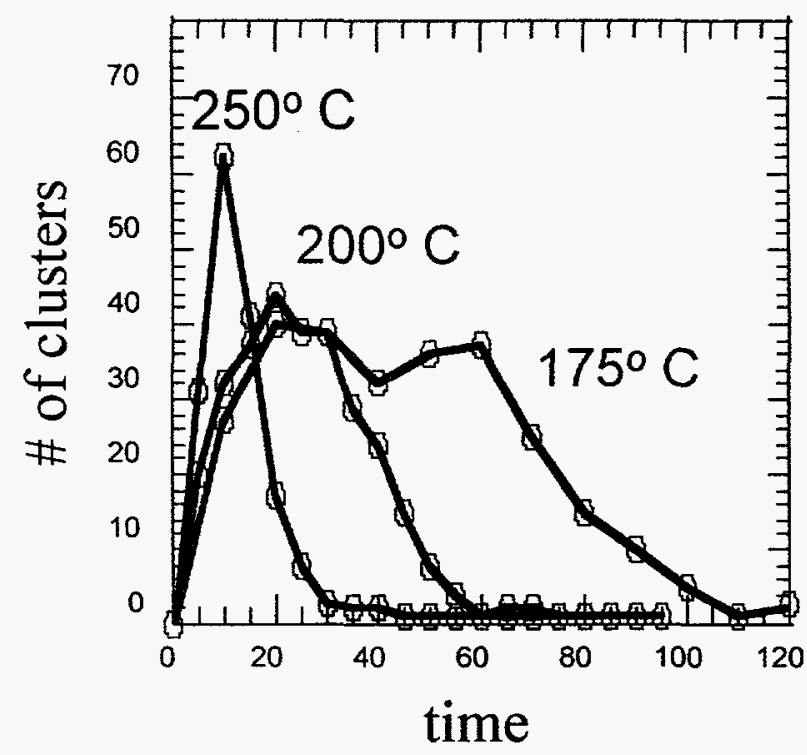

Figure 3. Number of individual clusters on the surface vs. time for temperatures indicated on figure. 
next section, we consider the effects of energetic differences between the clusters and the substrate that can explain the degree of smoothing after coalescence that we observe.

\section{Effect of energetic differences: surfaces and grain boundaries}

An alternative mechanism for enhancing the growth of clusters (and therefore the roughness) is the presence of an energetic difference between the substrates and the clusters. This induces a net current of atoms off the substrate and onto the clusters. This same energetic difference will also suppress the diffusion of atoms that land on the cluster onto the substrate. In addition, there may be energetic differences between the atoms at the boundaries between two clusters (grain boundaries) relative to atoms within the same cluster which can lower the rate of attachment at sites between the clusters and decrease the amount of smoothing after separate islands coalesce.

These energetic differences have been incorporated into Monte Carlo simulations of physical vapor deposition to model the growth process of $\mathrm{Al}$ on $\mathrm{SiO}_{2}$ (the details of these simulations will be published separately). The evolution of the surface morphology is shown in fig. 4 at three stages of growth ( $t=2,10$ and 30 seconds of simulated time). The upper panels correspond to the case where the grain boundary energy is zero, so that adatoms attaching at sites bridging the two clusters are bound as tightly as they would be within a single cluster. In this case, the boundary between clusters disappears when two clusters grow together. The lower panels correspond to a larger grain boundary energy so that adatoms are not bound as tightly at the grain boundary as they are at an equally coordinated site within a single cluster (the energy per bond across the grain boundary has been decreased from $0.25 \mathrm{eV}$ to $0.1 \mathrm{eV}$ for a random orientation). The grain boundaries after islands have coalesced are seen as the slightly darker gray regions in the film. The effect of the grain boundary energy is clearly seen after coalescence; when two clusters grow together with zero grain boundary energy, the surface rapidly smoothens due to adatoms attaching at the highly coordinated sites between the clusters. For the higher grain boundary energy, grooves remain between the grains when the grain boundary energy is non-zero. The

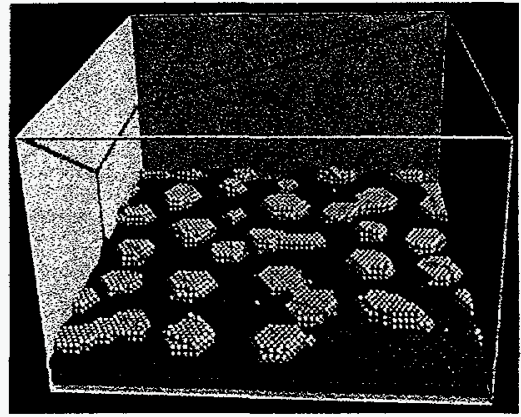

$$
\mathrm{t}=2 \mathrm{~s}
$$

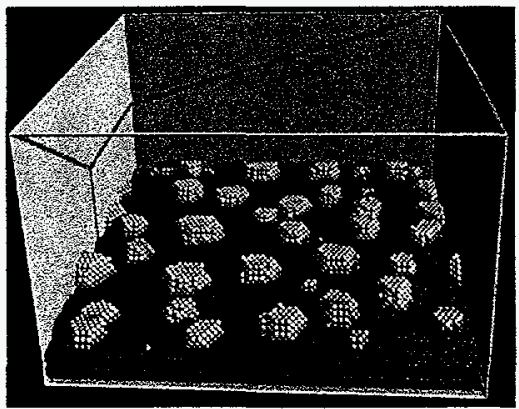

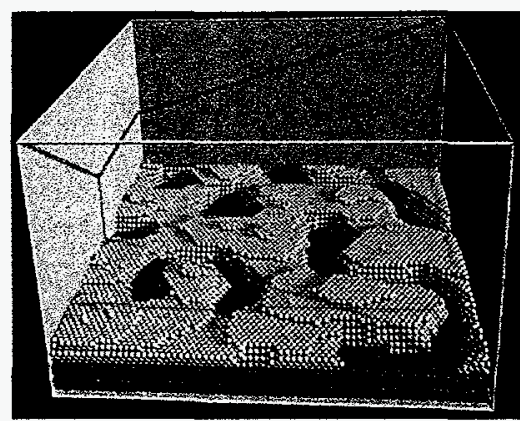

$\mathrm{t}=10 \mathrm{~s}$

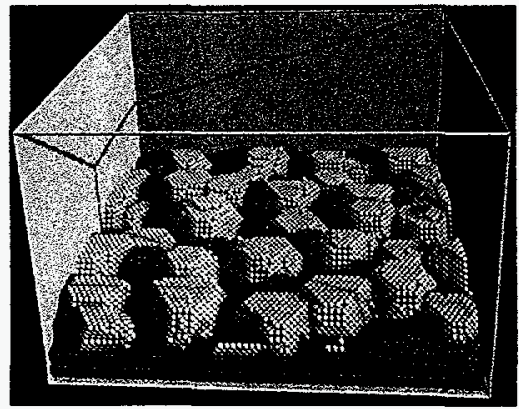

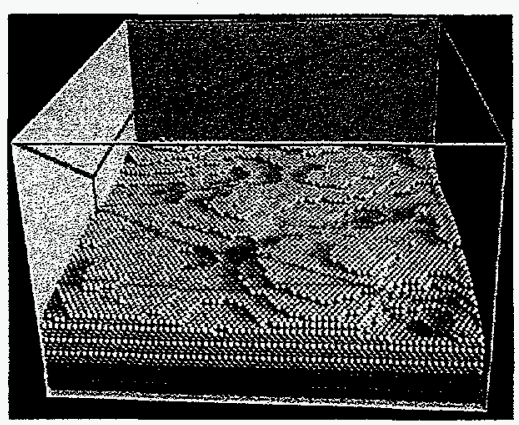

$$
\mathrm{t}=30 \mathrm{~s}
$$

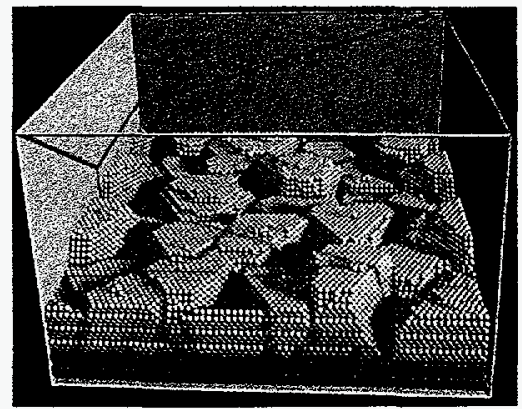

Figure 4. Simulated surfaces after 2,10 and $30 \mathrm{~s}$. Upper panels: no grain boundary energy; lower panels: with grain boundary energy. 


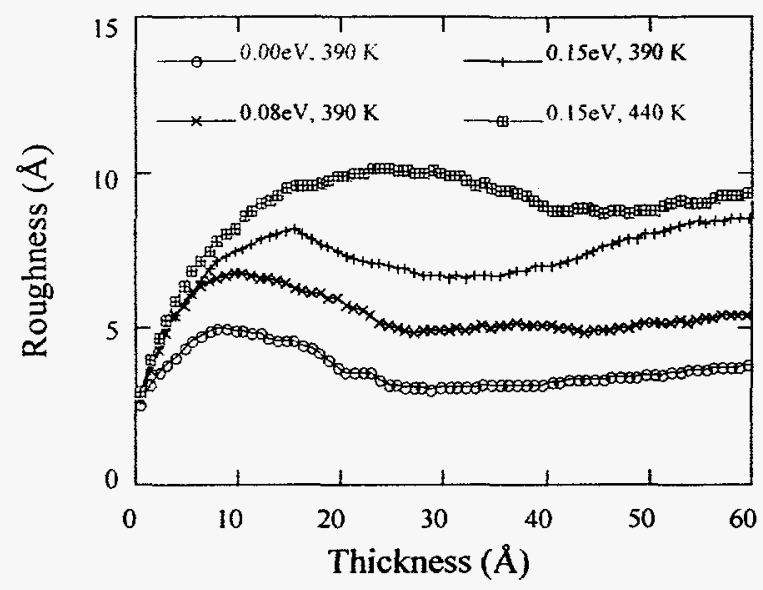

Figure 5. Evolution of surface roughness for various grain boundary energies.

roughening kinetics for these cases are shown fig. 5 . The energetic difference between the cluster surface and the substrate makes the roughness increase faster than the Poisson distribution case, similar to the case of CVD growth with preferential dissociation. The energetic differences between the surfaces also makes the simulated smoothing after coalescence more like the experimental case. After the substrate is completely covered, the barrier suppressing diffusion from the cluster to the substrate is no longer present and interlay transport can occur to smoothen the surface. However, the presence of a grain boundary energy can suppresses the smoothing that occurs after coalescence. With non-zero grain boundary energy, the maximum roughness is greater and the long term roughness is greater than for the case of zero grain boundary energy.

\section{CONCLUSIONS}

Monte Carlo simulations enable the study of individual surface processes that contribute to surface roughening. In order to explain the rapid roughening that occurs during cluster nucleation, barriers to interlayer transport are no sufficient. Mechanisms that lead to preferential growth on clusters are required, such as preferential dissociation on the cluster or energetic differences between the cluster and substrate. Rapid smoothing can occur after coalescence if there is a barrier to diffusion from the cluster onto the substrate. Energetic effects at the grain boundaries between clusters can suppress this smoothening and increase the roughness of the surface.

\section{ACKNOWLEDGMENTS}

The authors thank Jerry Floro for many useful discussions. This work was supported by the United States Department of Energy under Contract DE-AC04-94AL85000. Sandia is a multiprogram laboratory operated by Sandia Corporation, a Lockheed Martin Company, for the United States Department of Energy.

\section{REFERENCES}

1. B.K. Kellerman, E. Chason, D.P. Adams, T.M. Mayer and J.M. White, Surf. Sci., in press.

2. E. Chason and B.W. Dodson, J. Vac. Sci. Technol. A9, (1991) 1545.

3. D.P. Adams, T.M. Mayer, E. Chason, B.K. Kellerman and B.S. Swartzentruber, Surf. Sci., in press.

4. Hanchen Huang, unpublished.

5. P.I. Cohen, G.S. Petrich, P.R. Pukite and G.J. Whaley, Surf. Sci. 216, (1989) 222. 